\title{
TGFbDNRII-transduced Autologous Tumor Infiltrating Lymphocytes
}

National Cancer Institute

\section{Source}

National Cancer Institute. T GFbDNRII-transduced Autologous Tumor Infiltrating

Lymphocytes. NCI Thesaurus. Code C111992.

A preparation of tumor infiltrating lymphocytes (TILS) that are transduced with a retroviral vector encoding a gene for a dominant-negative form of the transforming growth factor beta (T GFb) receptor, T GFbDNRII, with potential immunomodulating activity. Upon administration, the TGFbDNRII-transduced autologous TILs recog nize and kill tumor cells. The expression of T GFbDNRII allows for the TILs to be resistant to TGFb-mediated inhibition of T cell proliferation and activation, which allows optimal TIL activity. The immunosuppressant TGF-b is produced by tumor cells and plays a key role in the repression of the immune system. 